\title{
Genome assemblies of three closely related leaf beetle species (Galerucella spp.)
}

\author{
Xuyue Yang (D) , ${ }^{1}$ Tanja Slotte (D) , ${ }^{1}$ Jacques Dainat, ${ }^{2}$ and Peter A. Hambäck ${ }^{1, *}$ \\ ${ }^{1}$ Department of Ecology, Environment and Plant Sciences, Stockholm University, Stockholm 10691, Sweden and \\ ${ }^{2}$ Department of Medical Biochemistry Microbiology and Genomics, National Bioinformatics Infrastructure Sweden, Science for Life Laboratory, Uppsala University, \\ Uppsala 75237, Sweden \\ *Corresponding author: Department of Ecology, Environment and Plant Sciences, Stockholm University, Stockholm 10691, Sweden. Email: peter.hamback@su.se
}

\begin{abstract}
Galerucella (Coleoptera: Chrysomelidae) is a leaf beetle genus that has been extensively used for ecological and evolutionary studies. It has also been used as biological control agent against invading purple loosestrife in North America, with large effects on biodiversity. Here, we report genome assembly and annotation of three closely related Galerucella species: G. calmariensis, G. pusilla, and G. tenella. The three assemblies have a genome size ranging from 460 to $588 \mathrm{Mbp}$, with N50 from 31,588 to 79,674 kbp, containing 29,202 to 40,929 scaffolds. Using an ab initio evidence-driven approach, 30,302 to 33,794 protein-coding genes were identified and functionally annotated. These draft genomes will contribute to the understanding of host-parasitoid interactions, evolutionary comparisons of leaf beetle species and future population genomics studies.
\end{abstract}

Keywords: Galerucella calmariensis; Galerucella pusilla; Galerucella tenella; Coleoptera; leaf beetle

\section{Introduction}

Galerucella (Coleoptera: Chrysomelidae) is a leaf beetle genus that is distributed worldwide except in the Neotropics (Thomas et al. 2002). Some species have been used as biological control agents against invasive plants, and the host specificity and environmental impact of these species have attracted broad interest. The most common application is the introduction of G. calmariensis and G. pusilla from Europe to North America against the invasive wetland plant purple loosestrife (Lythrum salicaria). Since 1992, releases of Galerucella populations have been made in many states in the USA and the colonization appears to have been successful, leading to a dramatic decrease of L. salicaria populations (Blossey et al. 1994; Landis et al. 2003; McAvoy et al. 2016).

In addition to its application in biological control, Galerucella spp. has been widely investigated in both ecological (Pappers et al. 2002; Tanaka and Nakasuji 2002; Hori et al. 2006; Fors et al. 2016) and evolutionary (Ikonen et al. 2003; Stenberg and Axelsson 2008; Yang et al. 2020) studies. In particular, Galerucella spp. has been used to study ecological and evolutionary consequences of hostparasitoid interactions (Stenberg et al. 2007), mainly involving three closely related species (G. calmariensis, G. pusilla, and G. tenella) with similar life cycles and their shared wasp parasitoid (Asecodes parviclava) (Hambäck et al. 2013; Fors et al. 2016). The divergence of these three species is fairly recent: G. pusilla and G. calmariensis diverged around 77,000 years ago while G. tenella diverged around 400,000 years ago (Hambäck et al. 2013). G. pusilla and G. calmariensis share an exclusive host plant (L. salicaria), whereas G. tenella feeds primarily on Filipendula ulmaria and occasionally on other Rosaceae species. In all three species, adults in the study area overwinter until mid-May and then lay eggs on leaves or stems of their host plant. Larvae hatch after 12 weeks, pupate in late June to early July, and the adults emerge from the pupae by the end of July. The three species are attacked by the same endoparasitoid wasp A. parviclava, which lays one or more eggs in the beetle larvae. The successful wasp larvae kills the host, use it as food resources, and subsequently emerge during the following summer (Hansson and Hambäck 2013).

The demography, host searching behavior, and immunology have previously been addressed in several Galerucella species (Zheng et al. 2008; Fors et al. 2015; Yang et al. 2020), but no genome assemblies of Galerucella species are currently available. The closest related species that has an available genome assembly is ragweed leaf beetle (Ophraella communa), which also belongs to the leaf beetle (Chrysomelidae) family (Bouchemousse et al. 2020). Here, we report de novo genome assemblies for G. calmariensis, G. pusilla, and G. tenella. We performed computational annotation, assigned gene ontology to functional proteins, and performed ortholog cluster analysis between the three species. These draft genomes will be useful for understanding the mechanisms underlying beetle interactions with parasitoid and plant use, and for future population genomics studies (McKenna 2018).

\section{Materials and methods DNA extraction and sequencing}

Larvae samples of G. pusilla and G. calmariensis were collected in

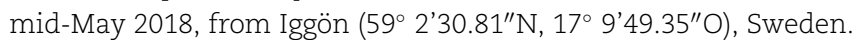


G. tenella samples were collected in mid-May 2018, from Södersjön (5951'8.72" N, $\left.18^{\circ} 6^{\prime} 26.59^{\prime \prime} \mathrm{O}\right)$, Sweden. To reduce heterozygosity and bacterial contamination, we reared and inbred the beetles in the laboratory at room temperature for one generation and collected adults from the second generation for DNA extraction.

For each species, we extracted DNA from one individual using an adjusted version of the $10 \mathrm{X}$ Genomics sample preparation protocol "DNA Extraction from Single Insects" (https://assets.ctfas sets.net/an68im79xiti/3oGwQ5kl6UyCocGgmoWQie/768ae48be4f 99b1f984e21e409e801fd/CG000145_SamplePrepDemonstratedPro tocol_-DNAExtractionSingleInsects.pdf). DNA concentrations were measured with a Qubit 3.0 Fluorometer using the dsDNA HS Assay Kit (Thermo Fisher Scientific) and DNA integrity was assessed on an agarose gel stained with 2\% GelRed. 10X Genomics Chromium linked-read sequencing libraries were prepared and subsequently sequenced to yield paired-end 2x150 bp reads, on a HiSeq X platform at SciLifeLab (Stockholm, Sweden).

\section{Genome assembly and scaffolding}

Raw 10X genomics reads were checked for sequencing quality using FastQC v0.11.5 (Andrews 2010), and de novo assembled using the Supernova v2.1.0 (Weisenfeld et al. 2017) assembler. We then polished the draft assembly using purge_dups v1.0.1 (Guan et al. 2020) to remove haplotigs and heterozygous overlaps based on sequence similarity and read depth. Subsequently, assemblies were scaffolded using arcs v1.0.6 (Yeo et al. 2018) and links v1.8.6, with the -a parameter, which controls the maximum link ratio between two best contig pairs set to 0.7 (Warren et al. 2015). To remove sequence contamination from the assembly, we ran Kraken v2.0 (Wood and Salzberg 2014) against bacterial, archaeal, and viral domains, along with the human genome. We assessed the completeness of our polished genome assemblies assessed by Benchmarking Universal Single-Copy Orthologs v4.0.5 (BUSCO) (Simão et al. 2015) from OrthoDB v9.1 (Zdobnov et al. 2017) using Endopterygota as the taxonomic database.

\section{Gene annotation and phylogenetic analysis}

We first assessed the repeat content of our genome assemblies and created a specific repeat library using RepeatModeler v1.0.11 (Smit et al. 2010b) for each genome assembly. Based on the repeat library, identification of repeat sequences in the genome was performed using RepeatMasker v3.0.9 (Smit et al. 2010a) and RepeatRunner (Yandell 2006) with default settings. RepeatRunner is a program that integrates RepeatMasker with BLASTX (Altschul et al. 1997), allowing the analysis of highly divergent repeats and identifications of divergent protein-coding portions of retro-elements and retroviruses.

Gene annotation was performed using the MAKER package v3.01.02 (Holt and Yandell 2011). First, for each genome, we generated one initial evidence-based annotation using both protein and transcriptome data sources. Protein databases came from the UniProt Swiss-Prot database (downloaded on 2019-11; 561,356 proteins) (Engler et al. 2020), as well a subset of manually selected proteins (uniport request: taxonomy: "Coleoptera [7041]," existence: "Inferred from homology [3]," 161,853 proteins). In addition to protein resources, transcriptome data containing 57,255 transcripts from G. pusilla were used as evidence for all three genomes (Yang et al. 2020). Next, we used the candidate genes from the initial annotation to train two different $a b$ initio gene predictors: Augustus v3.3.3 (Stanke et al. 2006) and Snap v2013_11_29 (Korf 2004). Finally, an ab initio evidence-driven gene build was generated based on the initial evidence-based annotation and the $a b$ initio predictions. In addition, we used EVidenceModeler v1.1.1 (Haas et al. 2008), which allows the construction of gene models based on the best possible set of exons produced by the $a b$ initio tools, and chooses those most consistent with the evidence. Functional inference for genes and transcripts was performed using the translated CDS features of each coding transcript.

Each predicted protein sequence was run against InterProscan (Jones et al. 2014) in order to retrieve functional information from 20 different sources. In addition, Blastp v2.9.0 (Altschul et al. 1990) was performed against the complete Swiss-Prot/UniProt database (downloaded 2019-2011) with a maximum e-value cutoff of $1_{e^{-}-6}$ to assign putative functions to predicted proteins. tRNA has been predicted through tRNAscan v1.3.1 (Lowe and Eddy 1997).

To confirm the evolutionary relationships between the three Galerucella species and their position in the Chrysomelidae family, we reconstructed a species tree based on predicted protein sets from G. calmariensis, G. pusilla, and G. tenella, O. communa, and L. decemlineata using OrthoFinder v2.4.0, with default settings except using multiple sequence alignments (-M msa) to infer the species tree (Emms and Kelly 2015).

\section{Ortholog cluster analysis}

Identifying shared orthologous clusters allows the comparison of the function and evolution of proteins across closely related species. An ortholog cluster analysis was performed by comparing the three complete Galerucella protein sets with each other via OrthoVenn2 (Xu et al. 2019) with default settings of $E=1_{e}-5$ and an inflation value of 1.5 .

\section{Data availability}

Raw read data and final assemblies are available at the EMBLENA database under BioProject PRJEB44256. Supplementary material including annotations is available at figshare: https://doi. org $/ 10.6084 / \mathrm{m} 9$.figshare.c.5470650. Command-line arguments and scripts for this study are available at: https://github.com/ Pikayy/Galerucella.

\section{Results and discussion Genome assemblies}

Sequencing of the 10X genomics libraries yielded a total of 683.34 million read pairs, resulting in a sequencing depth above 110X for each species. Due to the low molecular weight of the input DNA (average size $<20 \mathrm{kbp}$ ), the initial de novo assembly from Supernova was highly fragmented, with N50 values of 49.884 kbp, $19.764 \mathrm{kbp}$, and $24.604 \mathrm{kbp}$ for G. calmariensis, G. pusilla, and G. tenella. Redundancy removal by purge_dups and arcs+links scaffolding dramatically improved N50 values of assemblies (see Supplementary Table S1 for the comparisons between assemblies). The decontamination process removed two contigs from $G$. calmariensis, one contig from G. pusilla and two contigs from G. tenella which matched the human database with a kmer length $>100 \mathrm{bp}$. Final assemblies for $\mathrm{G}$. calmariensis had a size $=588 \mathrm{Mbp}$, contained 39,255 scaffolds with a N50 $=79.674 \mathrm{kbp}$, final assemblies for $\mathrm{G}$. pusilla had a size $=513 \mathrm{Mbp}, 40,929$ scaffolds with a $\mathrm{N} 50=45,442 \mathrm{kbp}$ whereas final assemblies for $\mathrm{G}$. tenella has a size $=460 \mathrm{Mbp}, 29,202$ scaffolds with a N50=31,588 kbp (Table 1). Using 2124 BUSCO groups with endopterygota_odb10 database, we found $91.3 \%$ complete orthologs and only $4.0 \%$ missing orthologs in G. calmariensis, 85.3\% complete orthologs and 6.5\% missing orthologs for G. pusilla and $95.4 \%$ complete orthologs and $3.3 \%$ missing orthologs for $G$. tenella. Although the final assembly was 
Table 1 Summary of G. calmariensis, G. pusilla, and G. tenella reference genomes

\begin{tabular}{|c|c|c|c|c|c|c|c|}
\hline Species & $\begin{array}{l}\text { Assembly } \\
\text { size (Mbp) }\end{array}$ & $\begin{array}{l}\text { Number of } \\
\text { scaffolds }\end{array}$ & $\begin{array}{c}\text { Scaffold } \\
\text { N50 (kbp) }\end{array}$ & $\begin{array}{l}\text { Max scaffold } \\
\text { length (Mbp) }\end{array}$ & $\begin{array}{l}\text { Number } \\
\text { of Ns }\end{array}$ & GC (\%) & $\begin{array}{c}\text { BUSCO } \\
\text { (Complete \%) }\end{array}$ \\
\hline G. calmariensis & 588.27 & 39,255 & 79.674 & 1.307 & $37,346,600$ & 33.8 & $1941 / 91.3$ \\
\hline G. pusilla & 513.24 & 40,929 & 45.442 & 3.034 & $36,426,200$ & 33.7 & $1812 / 85.3$ \\
\hline G. tenella & 460.59 & 29,202 & 31.588 & 0.234 & $2,669,407$ & 33.6 & $2027 / 95.4$ \\
\hline
\end{tabular}

BUSCO score is based on the Endopterygota_db10 dataset.

still fragmented, the completeness of genome measured by BUSCO was satisfactory. The GC content of the three genomes ranged from 33.6 to $33.8 \%$, which is slightly higher than the GC content of the ragweed leaf beetle genome assembly (Bouchemousse et al. 2020).

The sizes of the genome assemblies of our three Galerucella species varied (460 to $588 \mathrm{Mbp}$ ) but is slightly smaller than the size of the colorado potato beetle (Leptinotarsa decemlineata) (642 Mbp) (Schoville et al. 2018) and the ragweed leaf beetle (774 Mbp) (Bouchemousse et al. 2020). Coleoptera is amongst the most diverse insect orders in terms of genome size, with an average genome size of $760 \mathrm{Mbp}$ and ranges from 160 to $5020 \mathrm{Mbp}$ (Gregory 2021). Within-genus variation in genome size is relatively small in these three assemblies compared with other Coleopteran species, possibly because of their close phylogenetic relationships and similarities in life cycle, food sources, and wasp enemies.

\section{Gene annotation and phylogenetic analysis}

RepeatMasker masked $48.55,46.65$, and $40.84 \%$ of the G. calmariensis, G. pusilla, and G. tenella genomes as repetitive elements. In addition, RepeatRunner further masked approximately 1\% of each genome as repeats using MAKER TE as library (Supplementary Table S2).

The $a b$ initio evidence-driven annotation using the MAKER pipeline revealed $32,294,30,302$, and 33,794 potential proteincoding genes, accounting for 16.2, 17.7, and $19.1 \%$ of the whole genome of G. calmariensis, G. pusilla, and G. tenella respectively (Supplementary Table S3). For each species, 84 to $86 \%$ of proteincoding genes were assigned with a putative function, and 39-45\% had a GO annotation (Supplementary Table S4, functional annotations using InterProscan from 20 different sources). Blast against the UniProt/Swiss-Prot database predicted 15,046, 14,404, and 17,958 hits with unique gene names for G. calmariensis, G. pusilla, and G. tenella, respectively.

A maximum likelihood tree was built based on 1242 orthogroups shared between three Galerucella species and two other leaf beetles (Figure 1). The phylogenetic relationship is in accordance with previous studies generated by mitochondrial and nuclear genetic markers with high bootstrap support values for each branch (Hambäck et al. 2013; Bouchemousse et al. 2020).

\section{Ortholog cluster analysis}

The three protein sets of Galerucella species were compared to identify shared orthologous clusters using OrthoVenn2 (Figure 2). The complete protein sets contain 40,031 sequences from G. calmariensis, 37,514 sequences from G. pusilla and 44,200 sequences from $G$. tenella, corresponding to 20,665, 19,730, and 19,106 ortholog clusters, respectively.

Most annotated genes (12,372 orthogroups/48,398 proteins) were shared between the three species. Shared clusters between G. calmariensis and G. pusilla $(16,594)$ account for 80.3 and $84.1 \%$ of ortholog clusters in G. calmariensis and G. pusilla respectively whereas the shared regions of either G. calmariensis and G. pusilla

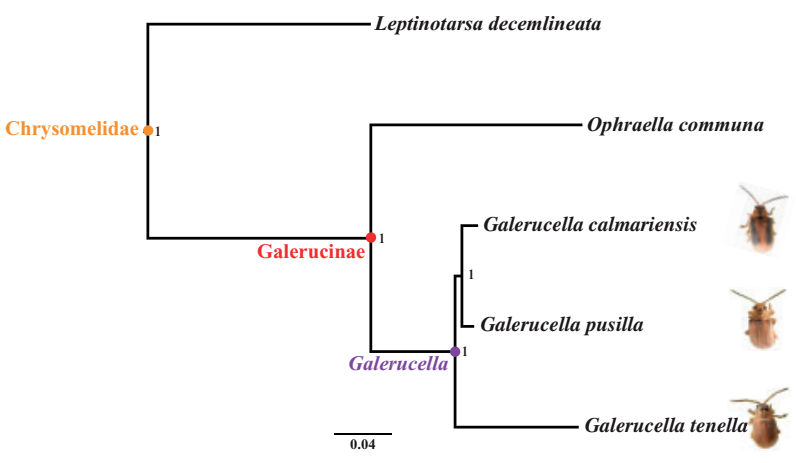

Figure 1 Rooted phylogenetic species tree of G. calmariensis, G. pusilla, G. tenella, and two leaf beetle species, O. communa and L. decemlineata (outgroup), derived from 1242 orthogroups using OrthoFinder. Branch labels indicate support values based on 1000 bootstrap replicates.

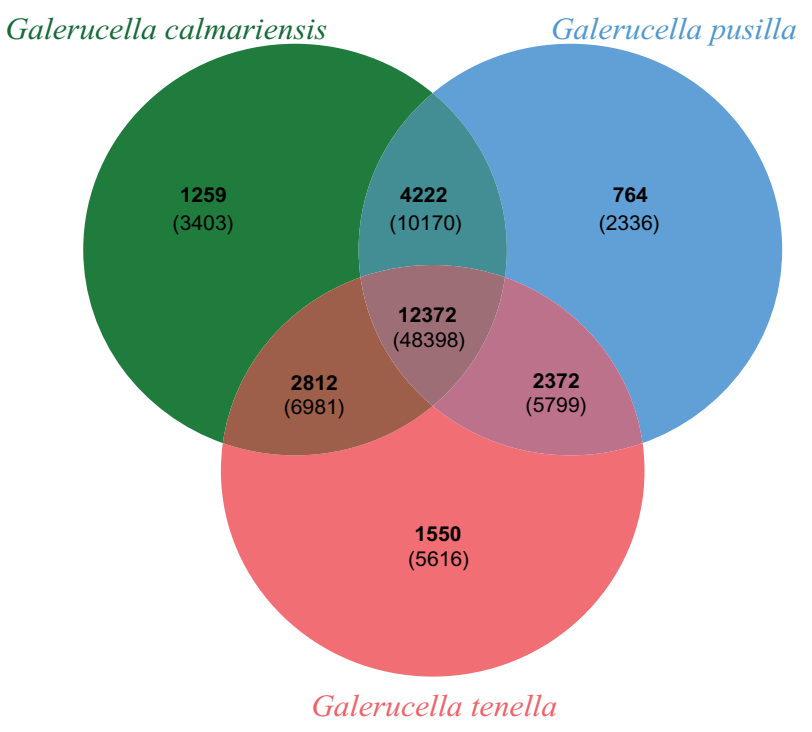

Figure 2 A Venn diagram of the orthologous gene clusters among the three Galerucella species: G. calmariensis, G. pusilla, and G. tenella. The numbers of shared Ortholog clusters between species is indicated in the overlapping areas of the circles while the numbers of proteins corresponding to each cluster are underneath in parentheses.

with G. tenella account for less than $75 \%$ of their clusters. Ortholog clusters unique to a single species account for 6.09 , 3.87, and $8.11 \%$ of the entire cluster set for G. calmariensis, G. pusilla, and G. tenella, which indicates divergent regions between species (Ferguson et al. 2020). The inflated numbers of singleton clusters in G. tenella may be due to the high duplication levels in the genome, as BUSCO detected 33.1\% complete duplicated BUSCOs in G. tenella. Whether this is due to gene duplication or assembly error should be further investigated. The duplication and fragmentation level detected by BUSCO are similar between G. calmariensis (1.8\% duplicated and $5.1 \%$ fragmented) and 
G. pusilla (1.2\% duplicated and $8.2 \%$ fragmented), however, G. calmariensis harbors a higher level of singleton clusters than G. pusilla.

\section{Conclusions}

Galerucella species play an important role as biological control agents as well as for ecological and evolutionary research. Here, we produced draft genome assemblies for three leaf beetles in the Galerucella genus, which are the first three genomes from the Galerucinae subfamily branch of the leaf beetle family. The genome sequencing of the three closely related beetles sharing a common wasp enemy also provides possibilities of understandings of food web and host-parasitoid interactions. In particular, comparing genomes of species with divergent immune resistance against parasitoid wasps may contribute to detecting essential genetic regions underlying host immunity and other potential traits participating in the arms race between host and parasitoids.

\section{Acknowledgments}

The authors would like to thank Remi-André Olsen at SciLifeLab Stockholm, Sweden for advice in genome assembly.

\section{Funding}

The authors would like to acknowledge support from Science for Life Laboratory, the National Genomics Infrastructure, NGI, and Uppmax for providing assistance in massive parallel sequencing and computational infrastructure. Computational analyses were enabled by resources provided by the Swedish National Infrastructure for Computing (SNIC) through Uppsala Multidisciplinary Center for Advanced Computational Science (UPPMAX) under project snic2017-7-233. SNIC is partially funded by the Swedish Research Council through grant agreement no. 2018-05973. This work was supported by grant VR-2015-4232 (to P.A.H.) from the Swedish Research Council.

\section{Conflicts of interest}

The authors declare that they have no competing interests.

\section{Literature cited}

Altschul SF, Madden TL, Schäffer AA, Zhang J, Zhang Z, et al. 1997. Gapped BLAST and PSI-BLAST: a new generation of protein database search programs. Nucleic Acids Res. 25:3389-3402.

Andrews S. 2010. FastQC: A Quality Control Tool for High Throughput Sequence Data. Cambridge: UK Babraham Inst.

Blossey B, Schroeder D, Hight SD, Malecki RA. 1994. Host specificity and environmental impact of two leaf beetles (Galerucella calmariensis and G. pusilla) for biological control of purple loosestrife (Lythrum salicaria). Weed Sci. 42:134-140.

Bouchemousse S, Falquet L, Müller-Schärer H. 2020. Genome assembly of the ragweed leaf beetle: a step forward to better predict rapid evolution of a weed biocontrol agent to environmental novelties. Genome Biol Evol. 12:1167-1173.

Emms DM, Kelly S. 2015. OrthoFinder: solving fundamental biases in whole genome comparisons dramatically improves orthogroup inference accuracy. Genome Biol. 16:14.
Engler JO, Lawrie Y, Gansemans Y, van Nieuwerburgh F, Suh A, et al. 2020. Genome Report: De novo genome assembly and annotation for the Taita white-eye (Zosterops silvanus). bioRxiv. doi: 10.1101/2020.03.06.980599.

Ferguson KB, Kursch-Metz T, Verhulst EC, Pannebakker BA. 2020. Hybrid genome assembly and evidence-Based annotation of the egg parasitoid and biological control agent trichogramma brassicae. G3 (Bethesda). 10:3533-3540.

Fors L, Liblikas I, Andersson P, Borg-Karlson A-K, Cabezas N. 2015. Chemical communication and host search in Galerucella leaf beetles. 33-45.

Fors L, Markus R, Theopold U, Ericson L, Hambäck PA. 2016. Geographic variation and trade-offs in parasitoid virulence. J Anim Ecol. 85:1595-1604.

Gregory TR. 2021. Animal genome size database. http://www. genomesize.com.

Guan D, McCarthy SA, Wood J, Howe K, Wang Y, et al. 2020. Identifying and removing haplotypic duplication in primary genome assemblies. Bioinformatics. 36:2896-2898.

Haas BJ, Salzberg SL, Zhu W, Pertea M, Allen JE, et al. 2008. Automated eukaryotic gene structure annotation using evidence modeler and the program to assemble spliced alignments. Genome Biol. 9:R7-R22.

Hambäck PA, Weingartner E, Ericson L, Fors L, Cassel-Lundhagen A, et al. 2013. Bayesian species delimitation reveals generalist and specialist parasitic wasps on Galerucella beetles (Chrysomelidae): sorting by herbivore or plant host. BMC Evol Biol. 13:92.

Hansson C, Hambäck PA. 2013. Three cryptic species in Asecodes (Förster) (Hymenoptera, Eulophidae) parasitizing larvae of Galerucella spp. (Coleoptera, Chrysomelidae), including a new species. JHR. 30:51-64.

Holt C, Yandell M. 2011. MAKER2: an annotation pipeline and genome-database management tool for second-generation genome projects. BMC Bioinformatics. 12:491-414.

Hori M, Ohuchi K, Matsuda K. 2006. Role of host plant volatile in the host-finding behavior of the strawberry leaf beetle, Galerucella vittaticollis Baly (Coleoptera: Chrysomelidae). Appl Entomol Zool. 41:357-363.

Ikonen A, Sipura M, Miettinen S, Tahvanainen J. 2003. Evidence for host race formation in the leaf beetle Galerucella lineola. Entomol Exp Appl. 108:179-185.

Jones P, Binns D, Chang H-Y, Fraser M, Li W, et al. 2014. InterProScan 5: genome-scale protein function classification. Bioinformatics. 30:1236-1240.

Korf I. 2004. Gene finding in novel genomes. BMC Bioinformatics. 5: 59.

Landis DA, Sebolt DC, Haas MJ, Klepinger M. 2003. Establishment and impact of Galerucella calmariensis L. (Coleoptera: Chrysomelidae) on Lythrum salicaria L. and associated plant communities in Michigan. Biol. Control. 28:78-91.

Lowe TM, Eddy SR. 1997. tRNAscan-SE: a program for improved detection of transfer RNA genes in genomic sequence. Nucleic Acids Res. 25:955-964.

McAvoy TJ, Kok LT, Johnson N. 2016. A multiyear year study of three plant communities with purple loosestrife and biological control agents in Virginia. Biol. Control. 94:62-73.

McKenna DD. 2018. Beetle genomes in the 21st century: prospects, progress and priorities. Curr Opin Insect Sci. 25:76-82.

Pappers SM, Van der Velde G, Ouborg JN. 2002. Host preference and larval performance suggest host race formation in Galerucella nymphaeae. Oecologia. 130:433-440.

Schoville SD, Chen YH, Andersson MN, Benoit JB, Bhandari A, et al. 2018. A model species for agricultural pest genomics: The 
genome of the Colorado potato beetle, Leptinotarsa decemlineata (Coleoptera: Chrysomelidae). Sci Rep. 8:1-18.

Simão FA, Waterhouse RM, Ioannidis P, Kriventseva EV, Zdobnov EM. 2015. BUSCO: assessing genome assembly and annotation completeness with single-copy orthologs. Bioinformatics. 31: 3210-3212.

Smit A, Hubley R, Green P. 2010a. RepeatMasker Open-3.0. Available from: https://www.repeatmasker.org.

Smit A, Hubley R, Green P. 2010b. RepeatModeler Open-1.0. Available from: https://www.repeatmasker.org/RepeatModeler.

Stanke M, Keller O, Gunduz I, Hayes A, Waack S, et al. 2006. AUGUSTUS: ab initio prediction of alternative transcripts. Nucleic Acids Res. 34:W435-W439.

Stenberg JA, Axelsson EP. 2008. Host race formation in the meadowsweet and strawberry feeding leaf beetle Galerucella tenella. Basic Appl Ecol. 9:560-567.

Stenberg JA, Heijari J, Holopainen JK, Ericson L. 2007. Presence of Lythrum salicaria enhances the bodyguard effects of the parasitoid Asecodes mento for Filipendula ulmaria. Oikos. 116:482-490.

Tanaka M, Nakasuji F. 2002. Dynamic interaction between a leaf beetle, Galerucella nipponensis, and an aquatic plant, Trapa japonica. II. Dispersal behavior of larvae. Popul Ecol. 44:1-6.

Thomas MC, Skelley PE, Frank JH. 2002. American Beetles, Volume II: Polyphaga: Scarabaeoidea Through Curculionoidea. Vol. 2. CRC Press.

Warren RL, Yang C, Vandervalk BP, Behsaz B, Lagman A, et al. 2015. LINKS: Scalable, alignment-free scaffolding of draft genomes with long reads. Gigascience. 4:15.
Weisenfeld NI, Kumar V, Shah P, Church DM, Jaffe DB. 2017. Direct determination of diploid genome sequences. Genome Res. 27: 757-767.

Wood DE, Salzberg SL. 2014. Kraken: ultrafast metagenomic sequence classification using exact alignments. Genome Biol. 15: R46.

Xu L, Dong Z, Fang L, Luo Y, Wei Z, et al. 2019. OrthoVenn2: a web server for whole-genome comparison and annotation of orthologous clusters across multiple species. Nucleic Acids Res. 47: W52-W58.

Yandell M. 2006. Comparative Genomics Library-RepeatRunner. Available from: https://www.yandell-lab.org/software/repeatrun ner.html.

Yang X, Fors L, Slotte T, Theopold U, Binzer-Panchal M, et al. 2020. Differential expression of immune genes between two closely related beetle species with different immunocompetence following attack by Asecodes parviclava. Genome Biol Evol. 12:522-534.

Yeo S, Coombe L, Warren RL, Chu J, Birol I. 2018. ARCS: scaffolding genome drafts with linked reads. Bioinformatics. 34:725-731.

Zdobnov EM, Tegenfeldt F, Kuznetsov D, Waterhouse RM, Simao FA, et al. 2017. OrthoDB v9. 1: cataloging evolutionary and functional annotations for animal, fungal, plant, archaeal, bacterial and viral orthologs. Nucleic Acids Res. 45:D744-D749.

Zheng F, Du Y, Wang Z, Xu J. 2008. Effect of temperature on the demography of Galerucella birmanica (Coleoptera: Chrysomelidae). Insect Sci. 15:375-380. 